\title{
Preoperative left atrial dysfunction and risk of postoperative atrial fibrillation complicating thoracic surgery
}

\author{
Tina Raman, MA, ${ }^{\mathrm{a}}$ Nancy Roistacher, MD, ${ }^{\mathrm{b}}$ Jennifer Liu, MD, ${ }^{\mathrm{b}}$ Hao Zhang, MD, ${ }^{\mathrm{a}}$ Weiji Shi, MS, ${ }^{\mathrm{c}}$ \\ Howard T. Thaler, $\mathrm{PhD},{ }^{\mathrm{c}}$ and David Amar, $\mathrm{MD}^{\mathrm{a}}$
}

\begin{abstract}
Objective: Postoperative atrial fibrillation complicating general thoracic surgery increases morbidity and stroke risk. We aimed to determine whether preoperative atrial dysfunction or other echocardiographic markers are associated with postoperative atrial fibrillation.
\end{abstract}

\begin{abstract}
Methods: In 191 patients who had undergone anatomic lung or esophageal resection, preoperative clinical and echocardiographic data were compared between patients with and without postoperative atrial fibrillation. Presence of postoperative atrial fibrillation lasting more than 5 minutes during hospitalization was detected using continuous telemetry or 12-lead electrocardiography. Maximal left atrial volume and indices of left atrial function were assessed.
\end{abstract}

Results: Patients with postoperative atrial fibrillation (33/191, 17\%) were older ( $71 \pm 5$ years vs $64 \pm 12$ years, $P<.0001)$, were taking $\beta$-blockers more often, had greater left atrial volume, had decreased left atrial emptying fraction, and had lower $\mathrm{E}^{\prime}$ and $\mathrm{A}^{\prime}$ septal velocities compared with patients without postoperative atrial fibrillation. The incidence of postoperative atrial fibrillation in patients with left atrial volume $32 \mathrm{~mL} / \mathrm{m}^{2}$ or greater was $37 \%(11 / 30)$ and greater than in those with left atrial volume less than $32 \mathrm{~mL} / \mathrm{m}^{2}(14 \%, 22 / 160, P=.002)$. Length of hospital stay was significantly increased in patients with postoperative atrial fibrillation compared with patients without $(P=.04)$. Older age was significantly associated with greater $\beta$-blocker use and left atrial volume and lower left atrial emptying fraction. On multivariate analysis, lower left atrial emptying fraction (odds ratio, 1.03 per unit decrement; 95\% confidence interval, $1.002-1.065 ; P=.04$ ) and preoperative use of $\beta$-blockers (odds ratio, 2.82; 95\% confidence interval, 1.18-6.77; $P=.02$ ) were the only independent risk factors associated with postoperative atrial fibrillation.

Conclusions: These data show that an echocardiogram before major thoracic surgery, increased use of preoperative $\beta$-blockers, and decreased left atrial emptying fraction were associated with postoperative atrial fibrillation. Echocardiographic predictors of left atrial mechanical dysfunction may prove clinically useful in risk stratifying patients in whom postoperative atrial fibrillation is more likely to develop and to benefit from prevention strategies aimed at mitigating atrial function before surgery. ( $\mathrm{J}$ Thorac Cardiovasc Surg 2012;143:482-7)

Atrial fibrillation (AF) occurs in $3 \%$ to $30 \%$ of patients after noncardiac thoracic surgery depending on the patient's age and whether an anatomic surgical resection is performed. ${ }^{1-3}$ The clinical symptoms, time of AF onset, and natural course of the arrhythmia are similar whether a patient has cardiac, thoracic, or other surgery. ${ }^{1-3}$ In virtually all studies of postoperative atrial fibrillation (POAF), increasing age has consistently been shown to be

From the Departments of Anesthesiology and Critical Care Medicine, ${ }^{\mathrm{a}}$ Medicine, ${ }^{\mathrm{b}}$ and Epidemiology and Biostatistics, ${ }^{\mathrm{c}}$ Memorial Sloan-Kettering Cancer Center, New York, NY.

Disclosures: Authors have nothing to disclose with regard to commercial support.

Received for publication June 6, 2011; revisions received July 12, 2011; accepted for publication Aug 24, 2011; available ahead of print Sept 28, 2011.

Address for reprints: David Amar, MD, Director of Thoracic Anesthesia, Memorial Sloan-Kettering Cancer Center, 1275 York Avenue, M-304, New York, NY 10021 (E-mail: amard@mskcc.org).

0022-5223/ $\$ 36.00$

Copyright (C) 2012 by The American Association for Thoracic Surgery doi:10.1016/j.jtcvs.2011.08.025 an independent preoperative risk factor regardless of type of surgery. ${ }^{1-5}$ Some studies have demonstrated that preexisting conditions, such as congestive heart failure and valvular heart disease, increase the risk for POAF in patients who do not undergo cardiac surgery. ${ }^{1}$ The mechanism responsible for POAF is not entirely clear but may represent a combination of the effects of altered sympathovagal balance, intraoperative trauma to autonomic fibers, and oxidative and inflammatory changes contributing to an enhanced susceptibility to reentrant arrhythmias after surgery. Identification of preoperative risk factors would allow for more targeted intervention in selected patients for whom preventive, preoperative antiarrhythmic therapy might be beneficial, while also reducing the exposure of those at a lower risk for POAF to potential drug-related toxicity and costs.

Recent literature has illustrated the predictive value of maximal left atrial volume (LAV) with regard to POAF, supporting the idea that left atrial enlargement reflects 


\author{
Abbreviations and Acronyms \\ $\mathrm{AF}=$ atrial fibrillation \\ $\mathrm{AUC}=$ area under the receiver operating \\ characteristic curve \\ $\mathrm{BNP}=$ brain natriuretic peptide \\ $\mathrm{CI}=$ confidence interval \\ LAEF $=$ left atrial emptying fraction \\ $\mathrm{LAV}=$ left atrial volume \\ $\mathrm{OR}=$ odds ratio \\ $\mathrm{POAF}=$ postoperative atrial fibrillation
}

increased left ventricular filling pressure and diastolic dysfunction. ${ }^{6,7}$ Controversy remains, however, as to which preoperative markers of atrial function will distinguish patients in whom POAF is most likely to develop. Thus, our goal was to focus on indices of left atrial function and POAF in a cohort of patients undergoing noncardiac thoracic surgery.

\section{MATERIALS AND METHODS}

With institutional review board approval, we retrospectively reviewed the charts of patients who had anatomic lung or esophageal resection of cancer with more than $80 \%(n=706)$ of the operations performed between June 2008 and August 2009. Patients who had preoperative echocardiography as part of their routine preoperative evaluation $(n=191)$ are the subjects of this study. Although there were no specific criteria applied to determine which patients had an echocardiogram before surgery, which was generally obtained by the surgeon or medical consultant, we compared patient characteristics (age, race, operation, smoking, hypertension, coronary artery disease, diabetes, and POAF rate) of the groups with or without an echocardiogram and found no significant differences. The primary end point of the study was the new onset of POAF lasting more than 5 minutes during hospitalization and detected by continuous telemetry or 12-lead electrocardiogram. Excluded were patients who were not in sinus rhythm before surgery or those with a history of paroxysmal AF, patients taking antiarrhythmic drugs, and patients who only had an exploratory thoracotomy or limited nonanatomic resection (eg, wedge), and therefore were at low risk for AF. Patients for whom echocardiographic images were not suitable for measurements of interest were also excluded $(n=3)$. Complete transthoracic echocardiograms and Doppler echocardiograms obtained for the 191 patients within 3 months before thoracic surgery were analyzed. Maximal LAV was calculated using the biplane area-length method, per the American Society of Echocardiography recommendation. ${ }^{8}$ On the basis of published data, a "gender neutral" cutoff of LAV $32 \mathrm{~mL} / \mathrm{m}^{2}$ or less was used as normal/mildly abnormal. ${ }^{6,7}$ To determine interobserver variability, LAV measurements were obtained by 2 independent investigators in $10 \%$ of the study population or 19 randomly selected cases. An estimated excellent intraclass correlation coefficient of 0.93 (95\% confidence interval $[\mathrm{CI}], 0.83-0.97)$ was obtained. Left atrial emptying fraction (LAEF) was calculated as follows: (maximal LAV-minimal LAV)/maximal LAV.

Postoperative pain relief was provided to all patients by continuous administration of epidural opioid (usually hydromorphone and bupivacaine). After an overnight stay in the postanesthesia care unit, patients were transferred to the thoracic surgical floor on postoperative day 1. Major postoperative cardiac or pulmonary complications were recorded throughout the hospital stay. An end point of the study was the new onset of AF for more than 5 minutes during hospital stay, detected by continuous telemetry (72-96 hours) or AF episodes requiring intervention because of symptoms or hemodynamic compromise. $\mathrm{AF}$ was defined by an irregularly irregular cardiac rhythm without clear P waves that was confirmed by 12-lead electrocardiogram.

The intraclass correlation coefficient and CI of LAV measurements between 2 observers was calculated on the basis of 1-way analysis of variance. To determine the difference of clinical characteristics and echocardiographic parameters between patients with and without POAF, univariate analysis was conducted using Student $t$ or chi-square test. Data are presented as mean value \pm standard deviation unless otherwise indicated. $P$ values were computed for each variable for all available data. Left atrial systolic volume, E-wave velocity, E/A ratio, E/E' septal ratio, and $\mathrm{E} / \mathrm{E}^{\prime}$ lateral ratio required log transformation to reduce skewness of the distributions. Multivariate logistic regression analysis was used to identify independent risk factors of POAF. Results were confirmed to agree with both forward and backward stepwise selection. This analysis was done first for echocardiographic parameters, and then clinical variables were added to the significant echocardiographic parameters. Variables with $P$ value of .05 or less by univariate analysis were included in the initial models (echocardiographic $\mathrm{n}=4$ and clinical $\mathrm{n}=2$ ). The area under the receiver operating characteristic curve (AUC) was calculated to measure the predictive power of identified parameters. Correlation between age and indices of atrial function was computed using Spearman correlation analysis. Cochran-Armitage trend test was used to assess the relationship between categories of LAEF and status of POAF. Statistical analysis was performed with SAS version 9.2 (SAS Institute Inc, Cary, NC).

\section{RESULTS}

The mean age for the 191 patients included in the study was $65.4 \pm 11.2$ years, and $53 \%$ were male. Patient characteristics and surgical data are presented in Table 1. POAF occurred in 33 of 191 patients $(17 \%)$ a median of 2 days (range, 0-6) after surgery. On univariate analysis, patients with POAF (70.5 \pm 5.3 years) were on average 6.2 years older (95\% CI, 3.6-8.8 years) than those without POAF $(64.4 \pm 11.8$ years $)(P<.0001)$ and proportionally taking more $\beta$-blockers before surgery $(P=.007$, Table 1$)$. The mean length of hospital stay was increased in patients with POAF $(9.1 \pm 5.0$ days) compared with those without POAF (7.9 \pm 7.4 days). The average length of hospital stay in patients with POAF was 1.29 times longer than those without POAF (95\% CI, 1.02-1.65) by $t$ test based on logarithm-transformed data $(P=.04)$.

Patients with POAF had greater maximal LAV, decreased LAEF, and lower $\mathrm{E}^{\prime}$ and $\mathrm{A}^{\prime}$ septal velocities compared with patients without POAF by univariate analyses $(P<.05$; Table 2 and Figure 1). By using a gender-neutral cutoff, ${ }^{8}$ the incidence of POAF in patients with LAV $32 \mathrm{~mL} / \mathrm{m}^{2}$ or greater was $37 \%(11 / 30)$ and significantly greater than in those with LAV less than $32 \mathrm{~mL} / \mathrm{m}^{2}(14 \%, 22 / 160$, $P=.002)$. There was a trend of greater minimal LAV and left ventricular diastolic filling pressure (as estimated by the $\mathrm{E} / \mathrm{E}^{\prime}$ septal ratio) in patients with POAF (Table 2). The groups did not differ in left ventricular ejection fraction. Consideration of 4 echocardiographic parameters with a $P$ value of .05 or less by univariate analysis in the 
TABLE 1. Patient and operative characteristics

\begin{tabular}{|c|c|c|c|}
\hline Variable & $\begin{array}{c}\text { Patients with } \\
\text { POAF }(n=33)\end{array}$ & $\begin{array}{l}\text { Patients without } \\
\text { POAF }(\mathbf{n}=158)\end{array}$ & $P$ value \\
\hline \multicolumn{4}{|l|}{ Preoperative } \\
\hline Age (y) & $70.5 \pm 5.3$ & $64.4 \pm 11.8$ & $<.0001$ \\
\hline Male gender, $\mathrm{n}(\%)$ & $21(64)$ & $78(49)$ & .14 \\
\hline White race & $29(88)$ & $144(91)$ & .52 \\
\hline Weight $(\mathrm{kg})$ & $79.6 \pm 16.2$ & $75.8 \pm 17.3$ & .25 \\
\hline Height $(\mathrm{cm})$ & $168.6 \pm 10.0$ & $167.5 \pm 10.1$ & .56 \\
\hline Body surface area $\left(\mathrm{m}^{2}\right)$ & $1.9 \pm 0.2$ & $1.8 \pm 0.2$ & .24 \\
\hline Preoperative heart rate (beats/min) & $74.0 \pm 15.2$ & $71.1 \pm 13.0$ & .27 \\
\hline Preoperative white blood cell count $(\mathrm{k} / \mu \mathrm{L})$ & $7.0 \pm 1.8$ & $6.9 \pm 2.2$ & .82 \\
\hline Diabetes, $\mathrm{n}(\%)$ & $6(18)$ & $17(11)$ & .23 \\
\hline Coronary artery disease, $\mathrm{n}(\%)$ & $11(33)$ & $39(25)$ & .30 \\
\hline Hypertension, $\mathrm{n}(\%)$ & $19(42)$ & $61(39)$ & .68 \\
\hline Mitral valve prolapse, $\mathrm{n}(\%)$ & $0(0)$ & $3(2)$ & 1.00 \\
\hline Congestive heart failure, $\mathrm{n}(\%)$ & $2(6)$ & $2(1)$ & .14 \\
\hline Chronic obstructive pulmonary disease, $\mathrm{n}(\%)$ & $4(12)$ & $6(4)$ & .07 \\
\hline Ex/current smoker, n (\%) & $26(79)$ & $110(70)$ & .29 \\
\hline Statin, n (\%) & $13(39)$ & $61(39)$ & 1.00 \\
\hline$\beta$-blocker, n (\%) & $14(42)$ & $32(20)$ & .007 \\
\hline Renin-angiotensin-aldosterone-system blocker, $\mathrm{n}(\%)$ & $10(30)$ & $38(24)$ & .51 \\
\hline Calcium channel blocker, n (\%) & $9(27)$ & $22(14)$ & .06 \\
\hline \multicolumn{4}{|l|}{ Operative } \\
\hline Total fluid input $(\mathrm{mL})$ & $2088 \pm 1642$ & $1872 \pm 1705$ & .19 \\
\hline Estimated blood loss & $286 \pm 192$ & $307 \pm 367$ & .74 \\
\hline Duration of operation (min) & $241 \pm 128$ & $218 \pm 106$ & .32 \\
\hline \multicolumn{4}{|l|}{ Surgical procedure } \\
\hline Esophagectomy, n (\%) & $8(24)$ & $38(24)$ & .82 \\
\hline Lobectomy, n (\%) & $20(61)$ & $99(63)$ & \\
\hline Pneumonectomy, n (\%) & $1(3)$ & $9(6)$ & \\
\hline Segmentectomy & $4(12)$ & $12(8)$ & \\
\hline \multicolumn{4}{|l|}{ Operation laterality (lung only) } \\
\hline Left, n $(\%)$ & $14(56)$ & $50(42)$ & .19 \\
\hline Right, n (\%) & $11(44)$ & $70(58)$ & \\
\hline
\end{tabular}

$P O A F$, Postoperative atrial fibrillation.

initial multivariate logistic regression analysis showed that lower LAEF (odds ratio [OR], 1.04 per unit decrement; $95 \%$ CI, $1.01-1.07 ; P=.005 ;$ AUC $=0.59$ ) was the only echocardiographic parameter significantly associated with POAF. Further multivariate logistic regression by adding 2 clinical characteristics with a $P$ value of .05 or less on univariate analysis showed that lower LAEF (OR, 1.03 per unit decrement; 95\% CI, 1.001-0.064; $P=.04)$ and preoperative use of $\beta$-blockers (OR, 2.82; 95\% CI, 1.18 $6.77 ; P=.02)$ were the only independent risk factors associated with POAF (Table 3). The AUC for this final model was 0.67 and moderately predictive of POAF and the Hosmer-Lemeshow statistic $(P=.09)$. Preoperative use of $\beta$-blockers was significantly associated with older age $(P=.0002$ by $t$ test). With LAEF and preoperative use of $\beta$-blockers in the model, age did not add further significant prognostic information $(P=.09)$. By Spearman correlation analysis, greater age was significantly associated with higher LAV ( $\rho=0.24, P=.001)$, lower $\mathrm{E}^{\prime}$ septal velocity $(\rho=-0.25, P=.008)$, and decreased LAEF $(\rho=-0.26$, $P=.001)$.

\section{DISCUSSION}

The main finding is that greater preoperative use of $\beta$-blockers and decreased LAEF were important risk factors for POAF in patients who had an echocardiogram before anatomic lung or esophageal resection. Older age was significantly associated with greater $\beta$-blocker use and LAV and lower LAEF. It is likely that greater preoperative use of $\beta$-blockers in this cohort was a surrogate marker of a trend in many institutions at the time these patients underwent operation to prophylactically administer these medications to reduce perioperative cardiovascular morbidity, rather than a cause and effect. For these reasons, we believe that older age, a known predictor of POAF, ${ }^{1-5}$ dropped out of the final multivariate model. Our patients had no clinical signs of 
TABLE 2. Echocardiographic parameters

\begin{tabular}{lccc}
\hline \multicolumn{1}{c}{ Parameter } & $\begin{array}{c}\text { Patients } \\
\text { with POAF } \\
(\mathbf{n}=\mathbf{3 3})\end{array}$ & $\begin{array}{c}\text { Patients } \\
\text { without POAF } \\
(\mathbf{n}=\mathbf{1 5 8})\end{array}$ & $\boldsymbol{P}$ value \\
\hline LV IDs $(\mathrm{cm})$ & $3.2 \pm 0.9$ & $3.0 \pm 0.6$ & .46 \\
LV wall mass $\left(\mathrm{g} / \mathrm{m}^{2}\right)$ & $85.4 \pm 29.5$ & $76.6 \pm 20.3$ & .11 \\
LV ejection fraction $(\%)$ & $61.6 \pm 12.6$ & $64.9 \pm 7.9$ & .16 \\
LA diastolic volume $\left(\mathrm{mL} / \mathrm{m}^{2}\right)$ & $29.2 \pm 9.8$ & $25.5 \pm 7.5$ & .048 \\
LA systolic volume $\left(\mathrm{mL} / \mathrm{m}^{2}\right)$ & $14.9 \pm 10.4$ & $10.6 \pm 5.1$ & .053 \\
E-wave velocity $(\mathrm{cm} / \mathrm{s})$ & $81.1 \pm 31.5$ & $74.7 \pm 17.5$ & .53 \\
A-wave velocity $(\mathrm{cm} / \mathrm{s})$ & $77.6 \pm 23.1$ & $81.5 \pm 22.2$ & .37 \\
E $^{\prime}$ septal velocity $(\mathrm{cm} / \mathrm{s})$ & $6.6 \pm 1.4$ & $7.6 \pm 2.6$ & .004 \\
E $^{\prime}$ lateral velocity $(\mathrm{cm} / \mathrm{s})$ & $8.7 \pm 3.0$ & $9.5 \pm 3.3$ & .2 \\
A $^{\prime}$ septal velocity $(\mathrm{cm} / \mathrm{s})$ & $7.9 \pm 3.0$ & $8.9 \pm 2.4$ & .04 \\
A $^{\prime}$ lateral velocity $(\mathrm{cm} / \mathrm{s})$ & $9.3 \pm 4.2$ & $10.3 \pm 3.8$ & .22 \\
E/A ratio & $1.2 \pm 0.8$ & $1.0 \pm 0.4$ & .43 \\
E/E' septal ratio & $13.1 \pm 6.6$ & $11.3 \pm 7.5$ & .06 \\
E/E' lateral ratio & $11.1 \pm 8.8$ & $8.9 \pm 5.1$ & .1 \\
DT (ms) & $230 \pm 64.3$ & $227 \pm 47.8$ & .8 \\
LA emptying fraction & $52.1 \pm 18.6$ & $59.6 \pm 11.0$ & .04 \\
TR jet & $23.8 \pm 8.7$ & $23.2 \pm 7.3$ & .73 \\
Estimated PA systolic pressure & $29.8 \pm 10.1$ & $28.2 \pm 8.2$ & .42 \\
\hline$D T$, Deceleration time; $I D s$, internal dimension during systole; $L A$, left atrium; $L V$, left \\
ventricle; $P A$, pulmonary artery; $P O A F$, postoperative atrial fibrillation; $T R$, tricuspid \\
regurgitation. & & & \\
& & &
\end{tabular}

congestive heart failure or evidence of significant valvular heart disease as a group but did show subtle or "subclinical" signs of left atrial distension (using LAV $\geq$ $32 \mathrm{~mL} / \mathrm{m}^{2}$ ) and mechanical dysfunction as risk factors for POAF. In our first prospective study examining the value of serial echocardiographic changes in 100 patients undergoing major pulmonary resection, only a mild to moderate increase in postoperative tricuspid regurgitation jet velocity was shown to significantly correlate with supraventricular tachydysrhythmias consisting mostly of

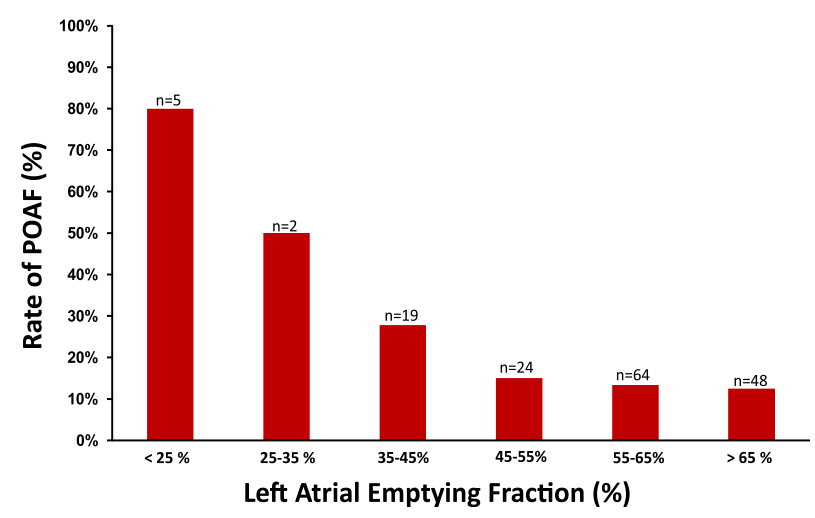

FIGURE 1. Relationship between left atrial emptying fraction and rate of POAF. Stratification of left atrial emptying fraction values into 6 groups ranging from an left atrial emptying fraction less than $25 \%$ to more than $65 \%$ indicated that the event rate of atrial fibrillation increased as left atrial emptying fraction decreased $(P=.005)$. POAF, Postoperative atrial fibrillation.
TABLE 3. Multivariate analysis of risk factors for postoperative atrial fibrillation

\begin{tabular}{lccc}
\hline & OR & $\mathbf{9 5} \%$ CI & $\boldsymbol{P}$ value \\
\hline$\beta$-blocker use & 2.82 & $1.18-6.77$ & .02 \\
LAEF, per unit decrement & 1.03 & $1.002-1.07$ & .04 \\
\hline
\end{tabular}

$L A E F$, Left atrial emptying fraction.

POAF. $^{2}$ This suggested that increased right heart pressure could be a contributing mechanism for POAF. However, none of the preoperative parameters, including left atrial size, by standard 2-dimensional measurements were predictive in that study. In contrast, newer echocardiographic methods (eg, integrated LAV and LAEF) have allowed us to better examine left atrial function in the current study. We have no clear explanation as to why patients with POAF were taking $\beta$-blockers more often than those without POAF and paradoxically had a higher incidence of POAF. Because the incidence of other comorbidities (eg, hypertension or coronary artery disease) did not differ between these groups, it is plausible that patients selected for this study may have had more preoperative testing (eg, baseline echocardiograms) in comparison with younger patients and perhaps as a result were given prophylactic $\beta$-blockers more often for perioperative cardiac protection.

Vaporciyan and colleagues ${ }^{9}$ showed that preoperative risk factors, such as increased age, male gender, and a history of congestive heart failure, arrhythmias, or peripheral vascular disease were all correlated with the development of POAF in a large database of patients undergoing noncardiac thoracic surgery. In a similar study of patients undergoing lung resection, Roselli and colleagues ${ }^{10}$ found that older age, male gender, paroxysmal AF, heart failure, clamshell incision, and right pneumonectomy were correlated with POAF. They also found that the majority of patients with POAF had concurrent cardiovascular, respiratory, or infectious complications, possibly reflecting an inflammatory role in the pathophysiology of POAF. Such variations in study outcomes are common among large epidemiologic studies and may reflect differences in patient characteristics, demographics surveyed, study sample size, and types of procedures. In previous work, we devised a clinical prediction rule and point score for POAF risk using the easily available preoperative clinical characteristics of age more than 55 years, male gender, and preoperative heart rate greater than 72 beats/min, with age as the most important risk factor. ${ }^{11}$ We examined whether inflammation contributed to POAF but could not demonstrate greater levels of C-reactive protein among patients with POAF by using serial measurements. ${ }^{12}$ However, we did find in another study that patients with POAF were more likely to have a higher white blood cell count on postoperative day 1 than those without POAF. ${ }^{13}$ In the search for serum marker correlates of POAF, it has been shown that subjects who had AF after 
esophageal or pulmonary resection had increased atrial production of brain natriuretic peptide (BNP). ${ }^{14,15}$ Cardinale and colleagues ${ }^{16}$ evaluated more than 400 patients undergoing thoracic surgery for lung cancer and showed that in patients with POAF, the pre- and postoperative $\mathrm{N}$-terminalproBNP values were significantly higher than in those patients without POAF. ${ }^{16}$ Taken together, these and our results suggest that the presence of subclinical left atrial dysfunction before surgery as measured by echocardiography or elevated BNP contribute to the electrical substrate necessary to initiate POAF in older patients undergoing major thoracic surgery. ${ }^{14-16}$

Unrelated to surgery, LAV has been shown to linearly correlate with increased left ventricular end-diastolic pressure and thus can be an indication of diastolic dysfunction. ${ }^{17}$ One of the original studies looking at patients with preexisting mitral valve disease showed that a left atrial dimension greater than $40 \mathrm{~mm}$ was significantly associated with the onset of $\mathrm{AF} .{ }^{18}$ Chronic exposure to increased ventricular filling pressures can cause increased atrial wall tension and stretch. ${ }^{19,20}$ Various mechanisms have been proposed to explain this phenomenon, including an increase in atrial effective refractory period because of myocyte stretch and myocardial fibrosis. ${ }^{21}$ In the cardiac surgical population, Roshanali and colleagues $^{22}$ showed that patients with POAF had significantly decreased preoperative A-wave transmitral flow velocity, increased LAV, and a prolongation of the interval from the onset of the $\mathrm{P}$ wave to the beginning of atrial systole, as measured at the lateral side of the left atrium. Benedetto and colleagues ${ }^{23}$ demonstrated that a larger left atrial area and peak atrial systolic mitral annular velocity (A velocity) $9 \mathrm{~cm} / \mathrm{s}$ or less were independent predictors of POAF in this population. ${ }^{23}$ Recent work by Nojiri and colleagues $^{24}$ showed that higher early transmitral velocity/mitral annular early diastolic activity was found to be significantly associated with POAF in patients who had undergone lobectomy for lung cancer resection. We found that patients undergoing noncardiac thoracic surgery who had POAF had significantly decreased mitral annular velocities $\left(\mathrm{E}^{\prime}\right)$, although we did not find any difference between the 2 groups with respect to the transmitral early peak velocity (E). Although the study by Nojiri and colleagues ${ }^{24}$ complements our work, they excluded patients undergoing segmentectomy, bilobectomy, or pneumonectomy and did not evaluate LAV or left atrial ejection or emptying fraction, which we found as more significant discriminators for POAF risk.

\section{Study Limitations}

Although this analysis was retrospective, this population represents a cohort of patients whose clinical data were collected and recorded at regular intervals. Manual measurement of LAV is dependent on accurate circumferential tracing of the left atrial chamber and computation of the long axis. This is subject to some level of variability based on the observer. However, we did find a high level of agreement in left atrial measurements between 2 independent investigators in a subset of randomly selected studies. The current study with 191 patients and 33 events (POAF) was of adequate sample size to identify several predictors that were significant on univariate analysis. However, the overall predictive accuracy is moderate $(\mathrm{AUC}=0.67)$, and some of the potential predictor variables are correlated with each other. Thus, only the strongest predictors remained in the final multivariable model. The model was not overfitted. A larger study would have to be done to confirm or rule out any additive predictive value from variables that in this study were only moderately significant on univariate analysis or to explore other clinical risk factors, such as race or cancer stage. $^{25}$

\section{CONCLUSIONS}

In a cohort of patients undergoing thoracic surgery who also had preoperative echocardiography, we found that greater use of preoperative $\beta$-blockers and indices of left atrial mechanical dysfunction were jointly associated with POAF occurrence. It is likely that greater preoperative use of $\beta$-blockers in this cohort was a surrogate marker of a trend in many institutions at the time these patients underwent operation to prophylactically administer these medications to reduce perioperative cardiovascular morbidity, rather than a cause and effect. Our echocardiographic findings are important in our efforts to better understand the pathophysiology of POAF. The use of these parameters may help in identifying a subset of patients with the highest risk of POAF. We speculate that these patients would most benefit from prophylactic interventions aimed at reducing LAV or improving LAEF in addition to conventional antiarrhythmics other than $\beta$-blockers. The goal would be to decrease the incidence of POAF and the length of stay and related costs in patients undergoing thoracic surgery.

\section{References}

1. Polanczyk CA, Goldman L, Marcantonio ER, Orav EJ, Lee TH. Supraventricular arrhythmia in patients having noncardiac surgery: clinical correlates and effect on length of stay. Ann Intern Med. 1998;129:279-85.

2. Amar D, Roistacher N, Burt M, Reinsel RA, Ginsberg RJ, Wilson RS. Clinical and echocardiographic correlates of symptomatic tachydysrhythmias after non-cardiac thoracic surgery. Chest. 1995;108:349-54.

3. Maisel WH, Rawn JD, Stevenson WG. Atrial fibrillation after cardiac surgery. Ann Intern Med. 2001;135:1061-73.

4. Park BJ, Zhang H, Rusch VW, Amar D. Video-assisted thoracic surgery does not reduce the incidence of postoperative atrial fibrillation after pulmonary lobectomy. J Thorac Cardiovasc Surg. 2007;133:775-9.

5. Amar D, Zhang H, Leung DHY, Roistacher N, Kadish AH. Older age is the strongest predictor of postoperative atrial fibrillation. Anesthesiology. 2002;96:352-6.

6. Tsang TS, Barnes ME, Bailey KR, Leibson CL, Montgomery SC, Takemoto Y, et al. Left atrial volume: important risk marker of incident atrial fibrillation in 1655 older men and women. Mayo Clinic Proc. 2001;76:467-75. 
7. Osranek M, Fatema K, Qaddoura F, Al-Saileek A, Barnes ME, Bailey KR, et al. Left atrial volume predicts the risk of atrial fibrillation after cardiac surgery. $\mathrm{J} \mathrm{Am}$ Coll Cardiol. 2006;48:779-86.

8. Lang RM, Bierig M, Devereux RB, Flachskampf FA, Foster E, Pellikka PA, et al, Chamber Quantification Writing Group, American Society of Echocardiography's Guidelines and Standards Committee, European Association of Echocardiography. Recommendations for chamber quantification: a report from the American Society of Echocardiography's Guidelines and Standards Committee and the Chamber Quantification Writing Group, developed in conjunction with the European Association of Echocardiography, a branch of the European Society of Cardiology. J Am Soc Echocardiogr. 2005;18:1440-63.

9. Vaporciyan AA, Correa AM, Rice DC, Roth JA, Smythe WR, Swisher SG, et al. Risk factors associated with atrial fibrillation after noncardiac thoracic surgery: analysis of 2588 patients. J Thorac Cardiovasc Surg. 2004;127:779-86.

10. Roselli EE, Murthy SC, Rice TW, Houghtaling PL, Pierce CD, Karchmer DP, et al. Atrial fibrillation complicating lung cancer resection. J Thorac Cardiovasc Surg. 2005;130:438-44

11. Passman RS, Gingold DS, Amar D, Lloyd-Jones D, Bennett CL, Zhang H, et al. Prediction rule for atrial fibrillation after major noncardiac thoracic surgery. Ann Thorac Surg. 2005;79:1698-703.

12. Amar D, Zhang H, Heerdt PM, Park B, Fleisher M, Thaler HT. Statin use is associated with a reduction in atrial fibrillation after noncardiac thoracic surgery independent of C-reactive protein. Chest. 2005;128:3421-7.

13. Amar D, Zhang H, Heerdt PM, Park B, Fleisher M, Thaler HT. Leukocytosis and increased risk of atrial fibrillation after general thoracic surgery. Ann Thorac Surg. 2006;82:1057-61.

14. Jiang-Long Hou, Ke Gao, Mei Li, Jian-Yang Ma, Ying-Kang Shi, Yun Wang, et al. Increased $\mathrm{N}$-terminal pro-brain natriuretic peptide level predicts atrial fibrillation after surgery for esophageal carcinoma. World J Gastroenterol. 2008;14:2582-5.

15. Nojiri T, Maeda H, Takeuchi Y, Funakoshi Y, Kimura T, Maekura R, et al. Predictive value of B-type natriuretic peptide for postoperative atrial fibrillation following pulmonary resection for lung cancer. Eur J Cardiothorac Surg. 2010;37:787-91.
16. Cardinale D, Colombo A, Sandri MT, Lamantia G, Colombo N, Civelli M, et al. Increased perioperative N-terminal pro-B-type natriuretic peptide levels predict atrial fibrillation after thoracic surgery for lung cancer. Circulation. 2007;115: 1339-44.

17. Simek CL, Feldman MD, Haber HL, Wu CC, Jayaweera AR, Kaul S. Relationship between left ventricular wall thickness and left atrial size: comparison with other measures of diastolic function. J Am Soc Echocardiogr. 1995;8:37-47.

18. Henry WL, Morganroth J, Pearlman AS, Clark CE, Redwood DR, Itscoitz SB et al. Relation between echocardiographically determined left atrial size and atrial fibrillation. Circulation. 1976;53:273-9.

19. Tsang TS, Barnes ME, Gersh BJ, Bailey KR, Seward JB. Left atrial volume as a morphophysiologic expression of left ventricular diastolic dysfunction and relation to cardiovascular risk burden. Am J Cardiol. 2002;15:1284-9.

20. Alsaileek AA, Osranek M, Fatema K, McCully RB, Tsang TS, Seward JB. Predictive value of normal left atrial volume in stress echocardiography. J Am Coll Cardiol. 2006;47:1024-8.

21. Chen YJ, Chen SA, Tai CT, Wen ZC, Feng AN, Ding YA, et al. Role of atrial electrophysiology and autonomic nervous system in patients with supraventricular tachycardia and paroxysmal atrial fibrillation. J Am Coll Cardiol. 1998;32:732-8

22. Roshanali F, Mandegar MH, Yousefnia MA, Rayatzadeh H, Alaeddini F, Amouzadeh F. Prediction of atrial fibrillation via atrial electromechanical interval after coronary artery bypasses grafting. Circulation. 2007;116:2012-7.

23. Benedetto U, Melina G, Roscitano A, Ciavarella GM, Tonelli E, Sinatra R. Clinical utility of tissue Doppler imaging in prediction of atrial fibrillation after coronary artery bypass grafting. Ann Thorac Surg. 2007;83:83-8.

24. Nojiri T, Maeda H, Takeuchi Y, Funakoshi Y, Maekura R, Yamamoto K, et al. Predictive value of preoperative tissue Doppler echocardiographic analysis for postoperative atrial fibrillation after pulmonary resection for lung cancer. $J$ Thorac Cardiovasc Surg. 2010;140:764-8.

25. Onaitis M, D'Amico T, Zhao Y, O'Brien S, Harpole D. Risk factors for atrial fibrillation after lung cancer surgery: analysis of the society of thoracic surgeons general thoracic surgery database. Ann Thorac Surg. 2010;90:368-74. 